\title{
El futuro de la protección social en Europa
}

\author{
Prof. Dr. Fernando Manrique López \\ Catedrático de la Universidad de Deusto
}

\section{El futuro de la Protección Social}

El mantenimiento de un alto nivel de empleo y de protección social es uno de los objetivos fundamentales de la Comunidad y está explícitamente incluido entre las tareas enumeradas en el artículo 2 del Tratado de la Unión Europea. La protección social representa un componente fundamental y un rasgo distintivo del modelo europeo de sociedad.

\section{Comisión Parlamento Europeo y Comité Económico y Social}

Por primera vez la Comisión al Parlamento Europeo y el Comité Económico y Social, se han fijado como metas:

1. ${ }^{\circ}$ Mejorar la eficacia de los sistemas de subsidio de desempleo: incrementar los incentivos para que los desempleados busquen un trabajo, un verdadero seguro que promueva la aptitud para el empleo en lugar de mera indemnización por desempleo. Transición gradual y flexible hacia la jubilación a fin de mantener las oportunidades para que los trabajadores de edad avanzada permanezcan en el mercado de trabajo.

2. Adoptar las consecuencias de lo que representa el envejecimiento de la población europea.

Entre 1995 y el 2025, el número de personas de 60 años o más, que ascendían en 1995 a 77 millones, aumentará como mínimo en 29 millones y, como máximo, en 44 millones. El porcentaje de personas de edad avanzada respecto a la población en su conjunto pasará de 21 a 
aproximadamente un 30 por ciento. Los ingresos transferidos de los trabajadores a los pensionistas vendrán alcanzando un nivel tal que se pondrá en peligro su viabilidad.

El creciente número de personas de edad avanzada generará una mayor demanda de atención sanitaria y servicios sociales cuya financiación pondrá igualmente en dificultades a los Estados miembros.

Por otro lado se destaca que durante los últimos 20 años, el empleo femenino en la Unión ha pasado de 46 a 61 millones de trabajadoras, mientras que el número de empleos masculinos ha permanecido estable. La progresión de la participación de la mujer en el mercado de trabajo se mantendrá en los próximo años y representará un factor positivo para compensar el envejecimiento de la población activa. No obstante no bastará con que la mujer trabaje, pues será indispensable el cumplimiento de otro fin: tendrá que tener hijos.

Efectivamente los cada vez más bajos índices de natalidad registrados en muchos Estados miembros muestran claramente la necesidad de remodelar los sistemas de protección social.

En este sentido cabe resaltar que a comienzos de siglo, Angel Pulido Fernández, en Salud pública en España y ministerio social de las clases médicas (Madrid, 1990), calculaba la mortalidad madrileña en un 40 por 100. Llegó a decir que la Villa y Corte era la necrópolis de España. Igualmente Segismundo Moret, en la publicación filokrausista La ilustración Ibérica, daba datos de las aterradoras cifras que el señor Moret expuso en una notable conferencia y que parecen el tañido funeral de la campanas que doblan por los difuntos.

El contraste lo tenemos a fecha de hoy al contemplar las cifras de esperanza de vida al nacer. Como dato esencial cabe resaltar que, en 1996 nos aventaja y por cierto, por muy poco, Suecia, Francia, Holanda e Italia pudiendo significarse que aquel tétrico panorama de comienzos de siglo ha desaparecido.

Simultáneamente contemplamos una formidable caída en la natalidad. En 1976 nacieron 667.456 niños; en 1998, 361.930, con una rapidez sin igual nos hemos convertido en el país de menor natalidad.

Según la encuesta de fecundidad del INE en 1999 estamos en la media de 1,07 hijos nacidos vivos por mayor de 15 a 45 años. La más baja del mundo.

Se suele repetir hoy en día que la compensación vendrá del exterior. Efectivamente un formidable diferencial del PIB por habitante -Marruecos - tiene el 9 por ciento del español o Ecuador el 11 por ciento, convierte a España de nación emigrante en inmigrante.

Ahora bien, si los atendemos, la carga sobre el Estado de Bienestar será bastante notable. 
La comodidad de no tener hijos y el alto nivel del bienestar material, que elimina la oferta de brazos para ciertas tareas sociales se va a pagar con dureza, guste o no a los españoles. Estos cinco problemas - los movimientos poblacionales entre el campo y la ciudad, el del paro, el del prolongado y amplio envejecimiento con sus secuelas en mayor carga financiera en pensiones y prestaciones sanitarias, los de la caída de la natalidad, y los de los emigrantes-, afectan a la médula de nuestro ser nacional. Es intolerable acercarse a ellos con ignorancia o demagogia como ha señalado el profesor Velarde.

3. ${ }^{\circ}$ Frente a quienes sostienen una pretendida armonización se debe recordar que la Unión cuenta simplemente con un sistema de coordinación de los regímenes de Seguridad Social de los Estados miembros. La protección social de un considerable número de personas por tanto dependen, entera o parcialmente, del buen funcionamiento del sistema de coordinación referido.

Cada vez son más numerosos los trabajadores altamente cualificados que migran, para los cuales los regímenes complementarios de protección social, que no han sido coordinados a nivel europeo, revisten una especial importancia.

Ahora bien se debe resaltar que, para hacer la protección social más favorable al empleo, convendría abordar los siguientes puntos:

1) Hacia una fiscalidad y sistemas de protección social más favorables al empleo.

Las personas menos incitadas a encontrar un empleo son las víctimas de la trampa del desempleo: si a la pérdida de prestaciones se suma la carga fiscal, pocas son las ventajas que cabe esperar por traer una renta profesional a la familia. En estos casos los beneficiarios de prestaciones se encuentran verdaderamente atrapados en un callejón sin salida, sin motivación económica alguna para escapar.

La mayor parte de los sistemas de desempleo fueron diseñados originalmente para proporcionar una renta sustitutoria a las personas que se encontraban, supuestamente por un breve período de tiempo, sin empleo. En la mayoría de los casos, el nuevo empleo requeriría cualificaciones idénticas o similares a las del trabajo anterior. Actualmente, es esencial que las personas que buscan empleo estén dispuestas a adquirir nuevas y diversas cualificaciones.

Las cifras revelan que mientras más se prolongue la situación de desempleo, mayor es la dificultad para reincorporarse al mercado. 
Se trata, pues, de adoptar medidas que ayuden a los desempleados a adquirir nuevas cualificaciones y a actualizar las que ya poseen, en un marco de protección social que prevenga la pobreza y la exclusión del mercado de trabajo y de la sociedad en general.

2) Implantación de sistemas flexibles para gestionar la transición de la vida profesional a la jubilación.

La situación de los trabajadores de edad avanzada ha cambiado radicalmente en los últimos veinte años.

Se introdujeron mecanismos que permitían a los afectados dejar el empleo mucho antes de alcanzar la edad de jubilación. La reducción de la vida profesional era objeto de un amplio consenso implícito entre empresarios, trabajadores y el Estado.

Sin embargo, debido al constante aumento de la esperanza de vida y a la introducción en muchos Estados miembros de reformas encaminadas a elevar la edad de jubilación para aliviar así la presión ejercida sobre los sistemas públicos de pensiones, resulta esencial invertir la tendencia hacia la salida anticipada del mercado de trabajo.

Se tiene poca conciencia de los problemas que planteará la jubilación anticipada durante los próximos diez años. A largo plazo, la tendencia actual de sustituir a los trabajadores de edad avanzada por trabajadores jóvenes será insostenible.

3) Hacia la viabilidad de los sistemas públicos de pensiones.

La mayoría de los Estados miembros centran sus esfuerzos en garantizar la continuidad de los regímenes públicos de reparto. La más importante de las soluciones adoptadas consiste en encontrar mecanismos que permitan a las personas continuar su actividad laboral en lugar de acogerse a la jubilación anticipada, es decir elevar la edad real de jubilación.

Otra tendencia común consiste en la estrecha relación que se está forjando entre la cuantía de las pensiones y las cotizaciones totales efectuadas a lo largo de la vida activa.

Fomentar el desarrollo de regímenes complementarios de pensión sobre una base completamente capitalizada. En este sentido, los fondos de pensiones podrán convivir paralelamente con los sistemas públicos de reparto.

4. ${ }^{\circ}$ Satisfacer las nuevas necesidades de asistencia de las personas de edad avanzada.

La esperanza de vida ha aumentado, y así surgen nuevas necesidades por lo que respecta a las condiciones de vida en la etapa final de la vida 
de las personas y el envejecimiento de la población europea tendrá una incidencia particularmente fuerte sobre las necesidades de asistencia.

Los avances en el tratamiento de enfermedades graves, así como el aumento generalizado de la esperanza de vida, supone que un número cada vez mayor de personas vive sus últimos años en una situación de dependencia en cuanto a sus necesidades cotidianas. Hasta ahora, solían ser los familiares quienes se ocupaban de estos casos.

Asistimos pues a la aparición de una nueva rama de la protección social. Sin embargo, no es fácil conciliar la necesidad de cubrir estas nuevas necesidades de asistencia con el imperativo de contener los costes correspondientes.

Puede, por tanto pensarse que se planteará una creciente demanda de servicios sociales, cuya satisfacción ofrece inicialmente grandes posibilidades en la creación de empleo.

\section{Mejora de los servicios sanitarios.}

En los Estados miembros, el gasto total en salud varía entre algo más del $5 \%$ a casi el $10 \%$ del PIB. En términos absolutos, el gasto ha venido aumentando durante muchos años y en todos los Estados miembros se constata una creciente presión sobre sus servicios sanitarios. Por ejemplo, el gasto per cápita en atención sanitaria para las personas de edad avanzada es cinco veces superior al correspondiente a las personas en edad de trabajar. Se espera que la categoría de edad de 75 años y más aumente en casi un $50 \%$ en los próximos 20 años, lo que tendrá un efecto significativo sobre el gasto sanitario total. Esta evolución se debe no sólo al cambio demográfico, sino también a las crecientes expectativas respecto a las prestaciones que los sistemas sanitarios pueden y deben ofrecer.

Resulta altamente llamativo el que, desde otra instancia como es el Consejo, por primera vez se plantean consideraciones similares y, en tal sentido deben exponerse las

\section{Conclusiones del Consejo de 17 de diciembre de 1999}

Estas son las siguientes:

- Velar por que el desarrollo económico y el desarrollo social vayan de la mano, reconociendo enfáticamente que la organización y financiación de la protección social competen a los Estados miembros. 
- Modernización en el seguimiento e intercambio de información y de las experiencias habidas resultando conveniente debatir el futuro de la protección social a escala europea en las nuevas circunstancias.

-El Consejo resalta además la función de los interlocutores sociales en la modernización del proceso de protección social.

- Por lo demás, se coincide en que deben aprovecharse plenamente las oportunidades que ofrecen las nuevas tecnologías, y en particular las de la información.

-Finalmente se resalta que el origen de los estudios que se llevan a cabo debe fijarse en los trabajos de un grupo de funcionarios de alto nivel.

\section{Parlamento}

Es en tercer lugar, el que viene a refrendar la línea de actuación mareada por los anteriores órganos y ello se manifiesta por los principios aceptados por la Cámara que resultan ser los recogidos en las orientaciones generales para la política económica del ponente Giorgos KATIFORIS. En tal sentido aparece que el Parlamento señala que es posible reducir los impuestos en muchos Estados miembros. También aboga por revisar los sistemas de Seguridad Social para reducir la carga fiscal que afecta a la creación de empleo.

En base a ello pueden extraerse las siguientes conclusiones:

- La Eurocámara considera que para conseguir el pleno empleo hacen falta elevadas tasas de crecimiento - no inflacionistas y económicamente sostenibles-. Para lograr ese crecimiento se necesita a su vez un entorno favorable a la inversión y formar adecuadamente a los trabajadores, y para ello, el Parlamento aconseja la reducción de trabas y de costes a la creación de empresas y que se siga avanzando en la reforma estructural del mercado laboral. Igualmente, se aboga por más eficacia en los mercados de bienes, servicios, capital y trabajo.

- Sigue siendo aconsejable mantener actitudes razonables durante las negociaciones salariales para tener en cuenta la estabilidad de precios, los aumentos de productividad y la necesidad de mantener bajo control los costes laborales unitarios, incluso en el caso de reducciones del tiempo de trabajo.

- La política económica, social y de empleo son tres aspectos de una estrategia global de combinación de políticas y, por lo tanto se entiende que, la aplicación de la nueva estrategia fortalecerá 
asimismo las iniciativas en materia de política social y de empleo y las equiparará a los instrumentos de la política económica.

- El logro de una economía basada en el conocimiento presupone una mayor actividad en los ámbitos de la investigación y el desarrollo.

- Las perspectivas de envejecimiento de la población hacen necesario llevar a cabo una revisión radical de los regímenes de pensiones para que sigan disfrutando de solidez financiera y continúen prestando un servicio a la población sobre una base justa y equitativa.

- La estabilidad de precios y salarios y una buena gestión de las finanzas públicas son condiciones necesarias para que los mercados operen sin sobresaltos y la economía funcione correctamente, en particular en lo que se refiere a las actividades de ahorro e inversión.

- Una distribución justa, una actitud solidaria en situaciones de adversidad económica y la adopción de un enfoque social en la toma de decisiones cotidianas son ingredientes esenciales para el satisfactorio funcionamiento de una economía de mercado.

\section{Consejo Europeo Extraordinario de Lisboa}

- Se insiste por lo demás en las conclusiones sostenidas en el Consejo Europeo extraordinario de Lisboa y la necesidad de obtener una vuelta al pleno empleo.

-El rápido envejecimiento de la población ha de abordarse como un problema grave a medio plazo, adoptando soluciones basadas en un enfoque fundado en la cohesión social.

- Se insta a aquellos Estados miembros que aún tengan que llevar a cabo reformas estructurales a que aumenten la flexibilidad de los mercados de bienes, servicios y capital, así como del mercado laboral.

- Se reconoce que es posible reducir el nivel de imposición en muchos de los Estados miembros y que hay que revisar los sistemas de seguridad social a fin de reducir la carga fiscal con respecto a la creación de empleo.

\section{Consejo Unión Europea 28-III-2000}

- Se acepta el criterio de la Presidencia del Consejo Europeo de Lisboa consistente en el aumento de la tasa de empleo, pasando de una media de $61 \%$ al $70 \%$ de la población para el 2010 . 
- Se recuerda que no deben utilizarse las inversiones públicas para gestionar la demanda en la economía, ya que la experiencia enseña que ello puede ser contraproducente; se considera que las inversiones públicas deben llevarse a cabo cuando los beneficios de dichas inversiones para la sociedad en su conjunto sean mayores que los costes.

- Se muestra a favor de la observancia de actitudes razonables en el marco de las negociaciones salariales, teniendo en cuenta la estabilidad de los precios, los aumentos de la productividad y la necesidad de mantener bajo control los costes laborales unitarios, incluso en el caso de reducciones del tiempo de trabajo, y se hace un llamamiento a los interlocutores sociales para que desarrollen las negociaciones o reformas al respecto con un espíritu de imparcialidad y responsabilidad social.

A este respecto debe ahora exponerse la síntesis del Informe sobre la protección social en Europa 1999 que recoge lo siguiente:

El rápido avance hacia una unión económica y monetaria completa, la estrategia concertada para el empleo y la ampliación de la Unión tienen importantes repercusiones para la protección social y hacen que sea cada vez más una cuestión de interés común para los Estados miembros y, por tanto, una cuestión en relación con la exigencia de que debe haber una mayor cooperación a nivel europeo.

Se acepta que los Estados miembros son responsables de la organización y la financiación de la protección social. Sin embargo, los instrumentos de la política de coordinación y supervisión de la UE, como las orientaciones generales de política económica o el Pacto de Estabilidad y Crecimiento, constituyen asimismo un marco general para las políticas de empleo y protección social, al establecer objetivos de coordinación presupuestaria y proporcionar directrices para las políticas públicas. El objetivo de esta nueva iniciativa para las políticas sociales, que tiene en cuenta la evolución del contexto económico es la necesidad de una política general de coordinación.

Pueden así aceptarse como Objetivos cruciales a que deben atenerse los Sistemas de Prevención Social los siguientes:

- hacer que trabajar sea rentable y garantizar unos ingresos seguros;

- conseguir pensiones seguras y sistemas de pensiones viables;

— promover la inclusión social; y

- garantizar una atención sanitaria viable y de alta calidad. 
Incluir la postergación de la edad oficial de jubilación o el aumento del número de años de cotización que se requieren para tener derecho a pensión completa. La reducción del importe de la pensión en relación con los últimos salarios o una relación más estrecha entre cotizaciones y prestaciones y la creación de fondos especiales para financiar las transferencias futuras. Esto equivale: a añadir a los sistemas de reparto existentes, en los que las pensiones actuales se financien con las cotizaciones actuales, sistemas de «capitalización» en los que las cotizaciones actuales se fijan en relación con los derechos futuros.

$\mathrm{Y}$ es que efectivamente, mientras que actualmente la Unión Europea cuenta con una media de una persona de 65 años o más por cada cuatro personas en edad de trabajar (coeficiente de dependencia de la tercera edad), se prevé que este porcentaje pase a ser uno por cada tres en el año 2020 y de casi uno por cada dos en el año 2040. Contrariamente a las tendencias observadas en el pasado, es poco probable que el crecimiento del coeficiente de dependencia de la tercera edad se compense en gran parte por la continuada disminución del número de niños y jóvenes menores de 15 años, que, según se prevé será más lenta que la del número de personas en edad de trabajar. El coeficiente global de dependencia, que en los últimos 20 años ha disminuido ligeramente en la Unión Europea, recuperará el terreno perdido antes del año 2010, para aumentar luego considerablemente desde el actual coeficiente de cinco personas menores de 15 años, o de 65 años o más por cada diez personas en edad de trabajar, a seis personas dependientes por cada diez personas en edad de trabajar en el año 2025 y siete personas dependientes por cada diez personas en edad de trabajar en el año 2040.

\section{El perfil del gasto social}

La prestación de vejez y viudedad representan, con mucho, el capítulo más importante de gastos de la protección social, con una media del 43\% del total de los gastos de la Unión Europea en 1996. Es decir, un poco más del $12 \%$ del PIB; la asistencia sanitaria ocupa el segundo lugar, con un poco más del $21,5 \%$ del total, o sea, algo más del 6\% del PIB.

Los gastos vinculados a las prestaciones de invalidez y de desempleo presentan una importancia similar. Cada uno de los capítulos representan algo más del $8 \%$ del total de los gastos, aunque en 1996, en 9 de los 15 Estados miembros el importe de las prestaciones por discapacidad era superior al de las prestaciones de desempleo.

Los gastos vinculados a las familias y a los niños, incluidas las prestaciones de maternidad, y los servicios de cuidados a los niños y 
las prestaciones familiares, representan una media sólo ligeramente inferior a las prestaciones de incapacidad y desempleo, mientras que los gastos vinculados a los otros tres tipos de prestaciones, a saber, enfermedad, vivienda y exclusión social, en conjunto son de importancia parecida, con un promedio de alrededor del 7,5\% del gasto global.

Financiación del gasto social

Por término medio, en 1996 las cotizaciones oficiales financiaron un $63 \%$ de los gastos de la protección social, si bien el porcentaje varió desde dos tercios en Bélgica, los Países Bajos, Alemania, Italia y España, a justo algo más de la mitad en Luxemburgo, Suecia y Finlandia y sólo el 25\% en Dinamarca.

Los métodos de financiación tienen tendencia a cambiar lentamente. La importancia relativa de las cotizaciones sociales en el conjunto de la Unión disminuyó marginalmente entre 1990 y 1996.

Por otro lado, las cotizaciones sociales se convirtieron en una fuente más importante de financiación en Dinamarca (donde se incrementó el porcentaje del $13 \%$ al 25\%) y los Países Bajos (de un $59 \%$ a un 67,5\%), también en Finlandia, Bélgica y Austria aunque bien es cierto que no tan espectacularmente.

\section{Cambios de Política}

Los cambios introducidos los últimos años en los sistemas de protección social se han centrado en los mismos objetivos clave mencionados anteriormente, puestos de relieve en la Comunicación de la Comisión publicada a principios de 1999 .

Mientras que en la década de los años 90 los cambios introducidos en las prestaciones de desempleo se dirigieron principalmente a frenar el gasto de los presupuestos públicos, en los últimos años se ha observado una tendencia generalizada a asociar más estrechamente el complemento a los ingresos con medidas activas del mercado de trabajo, así como a instar a las personas beneficiarias de prestaciones a que encuentren un trabajo lo más pronto posible. En varios Estados miembros, incluidos Dinamarca, Alemania, los Países Bajos y el Reino Unido, se ha ampliado la definición de trabajo adecuado para una persona en situación de desempleo, a fin de incluir en ella aquellos empleos que éstos pueden realizar con un mínimo de formación, en vez de incluir únicamente los trabajos realizados.

En varios Estados miembros, las prestaciones por discapacidad se han utilizado como sustituto de las prestaciones de un desempleo más 
«aceptable», especialmente en el caso de las personas en situación de desempleo de larga duración. En los Países Bajos y Reino Unido se introdujeron reformas dirigidas a reforzar los procedimientos asociados con los exámenes médicos y ampliar la definición de los trabajos que la persona en cuestión podría realizar.

En otros Estados, como en Austria, Grecia e Italia, también se han restringido los requisitos para causar derecho a prestaciones, mientras que en Suecia, donde los gastos son comparativamente elevados, el sistema se encuentra en pleno proceso de reforma.

En cuanto a la pensión de jubilación anticipada cabe significar que también ha aumentado el número de personas que se acogen a la jubilación anticipada, por razones similares, en tanto en cuanto se arbitran como alternativas al problema de desempleo de larga duración.

Para evitar tal utilización irregular de las prestaciones sociales en Dinamarca, Austria e Italia, se ha aumentado el número de años de cotización necesarios para poder optar a una jubilación anticipada, mientras que en Alemania, se está aumentando progresivamente la edad mínima para poder optar a una pensión de esas características.

Igualmente cabe resaltar las soluciones introducidas en Austria y Alemania en donde los empresarios tienen derecho a una reducción de sus cotizaciones sociales si, para sustituir progresivamente a sus trabajadores, contratan a una persona que se encuentra en situación de desempleo.

\section{Prestaciones familiares}

La protección social por hijos de las familias, con menores a cargo, tiene tres objetivos principales: ayudar a las personas a sobrellevar los costes adicionales que supone tener hijos por medio de prestaciones y subsidios especiales; ayudar a las personas que dan a luz a través de subsidios de maternidad y permisos, (que cada vez se aplican en mayor grado a ambos progenitores); y proporcionar ayuda para el cuidado de los niños y de los miembros de la familia con discapacidad o de edad avanzada y dependientes.

En Alemania, las prestaciones por hijo aumentaron perceptiblemente a principios de 1999 y se prevé que vuelvan a aumentar en el año 2000, lo que, junto a las desgravaciones fiscales por hijo, supondrá un incremento de los ingresos familiares netos. En Luxemburgo, la asistencia se dirige más específicamente a las familias de rentas bajas. En Italia se introdujo una nueva prestación en 1999 para con las familias con más de tres hijos menores de 18 años.

El derecho de las personas con menores a cargo para obtener un permiso se ha ampliado o está en proceso de ampliación en muchos 
países de la Unión, incluidos Austria, Luxemburgo, Dinamarca, Bélgica, Irlanda, los Países Bajos, Italia, España y el Reino Unido.

En España, las reducciones fiscales en concepto de costes para el cuidado de los niños menores de tres años aumentaron considerablemente en 1998. En los Países Bajos, desde 1999, los padres de familias monoparentales que buscan trabajo o reciben formación, tienen derecho a un reembolso de los gastos ocasionados por el cuidado de los niños. En el Reino Unido, en octubre de 1999 se introdujo un nuevo crédito fiscal para el cuidado de los niños, incluidas las familias monoparentales.

Para lograr pensiones seguras y unos sistemas financieros viables se han adoptado medidas tales como el aumento de la edad oficial de jubilación o del número de años de cotización necesarios para tener derecho a la pensión completa, la reducción del importe de la pensión en relación con el salario recibido en el pasado o una relación más estrecha entre las pensiones y las cotizaciones, así como la creación de fondos especiales para financiar transferencias.

En España, la base para calcular los derechos de pensión se encuentra en un proceso progresivo de extensión. En Italia, se congelaron las pensiones más elevadas en 1998. En Alemania se ha modificado la base para indexar las pensiones, con lo que se han reducido eficazmente los gastos. Debe destacarse, en tal sentido, la reforma de 1999, cuyo objetivo consistía en reducir gradualmente las pensiones desde un $70 \%$ del salario medio neto a un $64 \%$.

En España se ha aumentado el importe de las cotizaciones de las personas que perciben unos ingresos medios y altos, sin que este aumento haya ido acompañado de un aumento correspondiente de futuros derechos.

En los Países Bajos, se ha creado un Fondo Especial al que se contribuye cada año con una cantidad para el incremento del gasto. En Francia, existe la propuesta de incrementar de 40 a 42,5 los años de cotización necesarios para tener derecho a la pensión completa $\mathrm{y}$, al igual que en España, se ha creado un Fondo para consolidar la financiación de las pensiones, siguiendo el ejemplo de Irlanda en la que, se creó en 1998.

\section{Asistencia Sanitaria}

En muchos países con un sistema nacional de Asistencia Sanitaria (España, Italia, Suecia, el Reino Unido e Irlanda), se han traspasado mayores responsabilidades de gestión de los servicios y reparto de los presupuestos a las autoridades regionales o locales, que están en situa- 
ción de evaluar las necesidades de forma más precisa. Además, se ha observado una tendencia generalizada a aumentar las aportaciones directas por los medicamentos y los tratamientos, incrementando los costes directos a cargo del consumidor, a fin de instarles a racionalizar el uso de los servicios y proporcionar, además, medios de financiación suplementarios. Esta evolución ha ido acompañada del establecimiento de listas de medicamentos autorizados y de la introducción de medicamentos genéricos, a fin de reducir el coste de las recetas médicas.

Según datos procedente de la OCDE, el porcentaje de gastos privados en los gastos totales de la Asistencia Sanitaria en la Unión Europea ha sufrido un ligero incremento en la década de los años 90, habiendo pasado de un $22 \%$ en 1990 a casi el $24 \%$ en 1997 , lo que representa un aumento de algo más del $30 \%$ de los gastos de protección social. La importancia del gasto privado en la Asistencia Sanitaria varía considerablemente entre los Estados miembros, desde un $40 \%$ del total de los gastos en Portugal y el $30 \%$ en Italia hasta alrededor del $15 \%$ en Suecia, el Reino Unido, Bélgica y Dinamarca y sólo el 8\% en Luxemburgo. En países como Italia, Finlandia y Suecia, las restricciones del gasto de la salud pública han conducido al crecimiento de servicios privados entre 1990 y 1997, ya sea directa o indirectamente, puesto que el incremento de las cotizaciones directas de los asegurados constituyó una de las medidas para reducir los gastos públicos.

\section{Esquema esencial de los planteamientos sostenidos en el seno de la Unión Europea}

\section{A. Aspectos fundamentales del cambio y la modernización:}

Protección social más favorable al empleo.

Fiscalidad más favorable al empleo.

Transformar el seguro de desempleo de modo que a su través se procure una aptitud para el empleo.

Necesidad de reestructurar los modalidades de financiación de la Seguridad Social.

Sistemas flexibles para gestionar la transición de la vida profesional a la jubilación.

La protección social como instrumento para promover la integración.

\section{B. Principios esenciales:}

Adaptar la protección social al envejecimiento demográfico.

Lograr la viabilidad de los sistemas públicos. 
Revisar el contexto del seguro para los regímenes complementarios.

Examen de las nuevas necesidades de asistencia de las personas de edad avanzada.

Facilitar la mejora de los servicios sanitarios.

Adaptar la protección social a la evolución de la participación de hombres y mujeres en la vida profesional: hacia una individualización de los derechos.

Mejorar la protección social de las personas que se desplazan dentro de la Unión.

\section{Conclusiones sobre el futuro de la protección social}

Se hace preciso mostrar el camino que debe emprender la modernización.

Es necesario presentar algunas propuestas concretas con vistas a recabar apoyo a nivel europeo.

Cada Estado miembro es responsable de la organización y de la financiación de su sistema de protección oficial.

La Unión Europea debe velar por la coordinación de los regímenes encaminándolos a fomentar la libre circulación.

La Unión Europea ha de promover la comprensión, perspectivas a largo plazo y desafíos comunes.

2. Resulta imprescindible tener en cuenta lo que se denomina factor de producción: costes y beneficios globales de los sistemas de protección social.

3. Los Sistemas de protección social deberán adjuntar medidas internas que sean más favorables al empleo que los que hasta la fecha se han aprobado transformando el seguro de desempleo en un sistema paliativo de un seguro proactivo que promueva la aptitud para el empleo. Deben además reducirse los costes laborales no salariales, siguiendo los dictados del Informe del Consejo Europeo de Amsterdam 1997 y Luxemburgo 1997, sin obviar la incidencia de la imposición fiscal sobre el empleo tal y como recogió el Consejo Europeo de Dublín.

4. Adaptación de la protección social al envejecimiento demográfico de las sociedades europeas: El envejecimiento ejerce considerable presión sobre los sistemas de pensiones. Las reformas que es preciso introducir a fin de garantizar la viabilidad de los sistemas públicos de pensiones complementarias, ha de hacerse en un contexto seguro para los regímenes complementarios de pensiones.

Es indispensable contemplar las nuevas necesidades de personas dependientes de edad avanzada al igual que la eficacia y la rentabilidad de los sistemas sanitarios, con arreglo a las estrategias de mercados. 
5. Medidas encaminadas a hacer compatibles el trabajo y la vida familiar.

6. Protección social de las personas que se desplazan en el interior de la Unión.

7. Diálogo continuo sobre las estrategias de modernización de los sistemas de protección social.

\section{Documentación bibliográfica}

Documento de la Comisión. Consejo de la Unión Europea, sobre la modernización y mejora de la protección Social en Europa 12-III-97.

Conferencia sobre la Financiación de la Protección Social, Helsinki, Finlandia. 22-23-XI-99.

Conclusiones del Consejo de la Unión Europea sobre intensificación de la Cooperación para mejora y modernización de la protección social 17-XII-99.

Conclusiones de la Presidencia del Consejo de Europa. Lisboa 22-III-2000.

Informe sobre la Protección Social en Europa del Consejo de la Unión Europea 28-III-2000.

Informe Katifaris. Parlamento Europeo 11-V-2000.

Informe y recomendaciones sobre política y economía de los Estados miembros. Parlamento Europeo 10-V-2000. 